\title{
A multimodal protocol utilizing liposomal bupivacaine rib blocks leads to opioid reduction in patients undergoing the Nuss procedure
}

\author{
Alicia L. Eubanks^, David F. Grabski, Jessica Pollack, Daniel E. Levin, Eugene McGahren, \\ Linda W. Martin, Jeffrey Gander \\ Department of Surgery, University of Virginia, Charlottesville, VA, USA \\ Contributions: (I) Conception and design: J Gander, LW Martin, E McGahren, DE Levin, DF Grabski, AL Eubanks; (II) Administrative support: \\ AL Eubanks, J Pollack; (III) Provision of study materials or patients: J Gander, E McGahren, DE Levin, LW Martin; (IV) Collection and assembly \\ of data: AL Eubanks, J Pollack; (V) Data analysis and interpretation: DF Grabski, AL Eubanks; (VI) Manuscript writing: All authors; (VII) Final \\ approval of manuscript: All authors. \\ Correspondence to: Jeffrey Gander, MD. UVA Pediatric Surgery, 5th Floor, 1204 W. Main St., Charlottesville, VA 22903, USA.
}

Email: JG9BR@hscmail.mcc.virginia.edu.

Background: A major challenge associated with the Nuss procedure for pectus excavatum repair is postoperative pain control. Early Recovery Program (ERP) protocols for the Nuss procedure are becoming common, but there is a paucity of experience using liposomal bupivacaine (LB), a long-acting local anesthetic, for rib blocks in this setting. We investigated whether a protocol utilizing LB rib blocks decreased opioid use after the Nuss procedure while achieving equivalent pain control.

Methods: All adolescent patients undergoing the Nuss procedure at our institution between January 2013 and January 2021 were included. Patients were divided into a pre-intervention cohort $(\mathrm{n}=15)$, a transition cohort $(n=4)$, and a post-intervention cohort $(n=13)$. Patients in all groups received scheduled acetaminophen and non-steroidals postoperatively. The pre-intervention cohort received an opioid patientcontrolled analgesia (PCA) pump postoperatively, with a transition to oral opiates. The transition and postintervention cohorts received scheduled gabapentin in addition to intraoperative bilateral rib blocks with longer-acting local anesthetic. Rib blocks were performed using $0.25 \%$ Bupivacaine in the pre-intervention group. In the transition group, epinephrine $(1 \mathrm{mg} / \mathrm{kg})$ was added to $0.25 \%$ bupivacaine for the rib block. Following approval in patients aged $13-18$ years, $1.3 \% \mathrm{LB}(2.25 \mathrm{mg} / \mathrm{kg})$ was given for a rib block in the postintervention cohort.

Results: Demographic and clinical variables were equivalent in all groups. Post-intervention patients received $90 \%$ fewer opioids [median morphine equivalent (MME) $\mathrm{mg} / \mathrm{kg}$ ] compared to the pre-intervention cohort (0.8 vs. 8.2 MME $\mathrm{mg} / \mathrm{kg}, \mathrm{P}<0.0001)$, with no significant difference in pain scores between groups. Hospital length of stay was decreased among the intervention cohort ( $3 v s .4$ days, $\mathrm{P}=0.002$ ).

Conclusions: Significant decreases in opioid use and length of stay after the Nuss procedure were achieved by the implementation of a multimodal ERP for pain management, without increase in patient-reported pain scores.

Keywords: Nuss procedure; opioid reduction; intercostal block; liposomal bupivacaine (LB); Early Recovery Program (ERP)

Submitted Aug 10, 2021. Accepted for publication Oct 09, 2021.

doi: $10.21037 /$ jtd-21-1314

View this article at: https://dx.doi.org/10.21037/jtd-21-1314

\footnotetext{
$\wedge$ ORCID: 0000-0003-2141-8994.
} 


\section{Introduction}

The Nuss procedure is most often performed as a minimally invasive thoracoscopic approach to the repair of pectus excavatum (1). In this procedure, 1 or 2 preformed metal bars are placed within the chest wall to elevate the sternum and correct the deformity. A major challenge associated with this procedure is postoperative pain control. In addition to incisional pain, patients experience muscular and neuropathic pain associated with the stretching of the intercostal musculature that persists well beyond the operation (2). Poor pain control has been associated with increased patient and family anxiety, delay in initiation of physical therapy, and prolonged hospital stay (3). Historically the placement of a thoracic epidural for pain control was considered the standard of care at many programs (4). However, this has fallen out of favor due to the incidence of rare neurologic complications $(5,6)$ and the increased length of stay associated with epidural analgesia (3). More recently, many institutions favor the use of patient-controlled analgesia (PCA) with opioid medications (7). While opioids are effective, excessive use can have numerous adverse effects including nausea/ vomiting, constipation, urinary retention, and respiratory depression, as well as the potential for long term opioid dependence (8). As such, there is a trend at our institution and others toward alternative methods of multimodal pain control that minimize opioid administration. In thoracic surgery, there has been a major practice shift towards longacting rib blocks with liposomal bupivacaine (LB) $(9,10)$. There is a growing interest in the role of loco-regional anesthesia as an adjunct to a multimodal perioperative pain control strategy for the Nuss procedure. Methods previously investigated include paravertebral blocks (11), intercostal rib blocks with short-acting local anesthetic $(12,13)$, and intercostal nerve cryoablation (INC) (14-18). However, there is very little published in the literature with regards to the use of long-acting local anesthetic for rib blocks during this procedure in the adolescent population. The purpose of our study was to determine whether a new multimodal Early Recovery Program (ERP) utilizing rib blocks with LB for pain control decreased opioid use after the Nuss procedure. We hypothesized that compared to historical controls, patients managed in the setting of an ERP would have equivalent pain control with less opioid administration.

We present the following study in accordance with the TREND reporting checklist (available at https://dx.doi. org/10.21037/jtd-21-1314).

\section{Methods}

An opioid reduction quality improvement initiative (described below) was rolled out in all patients undergoing a Nuss procedure at our institution over the calendar year of 2018. Patients in the transition cohort (Jan-Dec 2018) and patients in the post-intervention cohort (Jan 2019-Jan 2021) were prospectively followed. For a historical control, a retrospective review of operative logs was undertaken to identify all patients that underwent a Nuss procedure at our institution from Jan 2013-Dec 2017. Three patients were excluded from the final analysis due to age $>21(n=1)$, reoperation for second bar placement $(\mathrm{n}=1)$, and incomplete data in the medical record $(n=1)$. Thirty-two patients were included in the study: 15 in the pre-intervention cohort, 4 in the transition cohort, and 13 in the post-intervention cohort.

Pre-operatively all patients underwent cardiopulmonary evaluation with electrocardiogram (EKG), echocardiogram, and CT scan or MRI to calculate Haller Index and degree of compression on intrathoracic structures, as well as metal allergy testing. Additionally, select patients had pulmonary function testing. Intra-operatively, all patients underwent a general anesthetic for the procedure. The medications and doses used for the anesthetic were at the discretion of the anesthesiologist and were not standardized across patients, nor considered part of our post-operative opioid reduction quality improvement intervention.

Table 1 describes the differences in perioperative management in the pre-intervention, transition, and postintervention cohorts. In the pre-intervention group, patients were given a rib block by palpation and subcutaneous infiltration with $0.25 \%$ Marcaine at the conclusion of the procedure. Postoperatively, patients in the pre-intervention (historical) cohort were administered scheduled acetaminophen and non-steroidal anti-inflammatory drugs (NSAIDs) (IV ketorolac then oral ibuprofen). They were prescribed an opioid PCA (morphine, fentanyl, or hydromorphone), and this was transitioned to oral opioids during the hospital stay.

Patients in the transition and post-intervention cohorts received pre-operative acetaminophen, celecoxib, and gabapentin. Patients in these groups also received intraoperative bilateral intercostal rib blocks at interspaces 5-9 bilaterally, performed by the operating surgeon. The method of rib block is important, and follows the technique 
Table 1 Perioperative protocols in pre-intervention, transition, and post-intervention cohorts

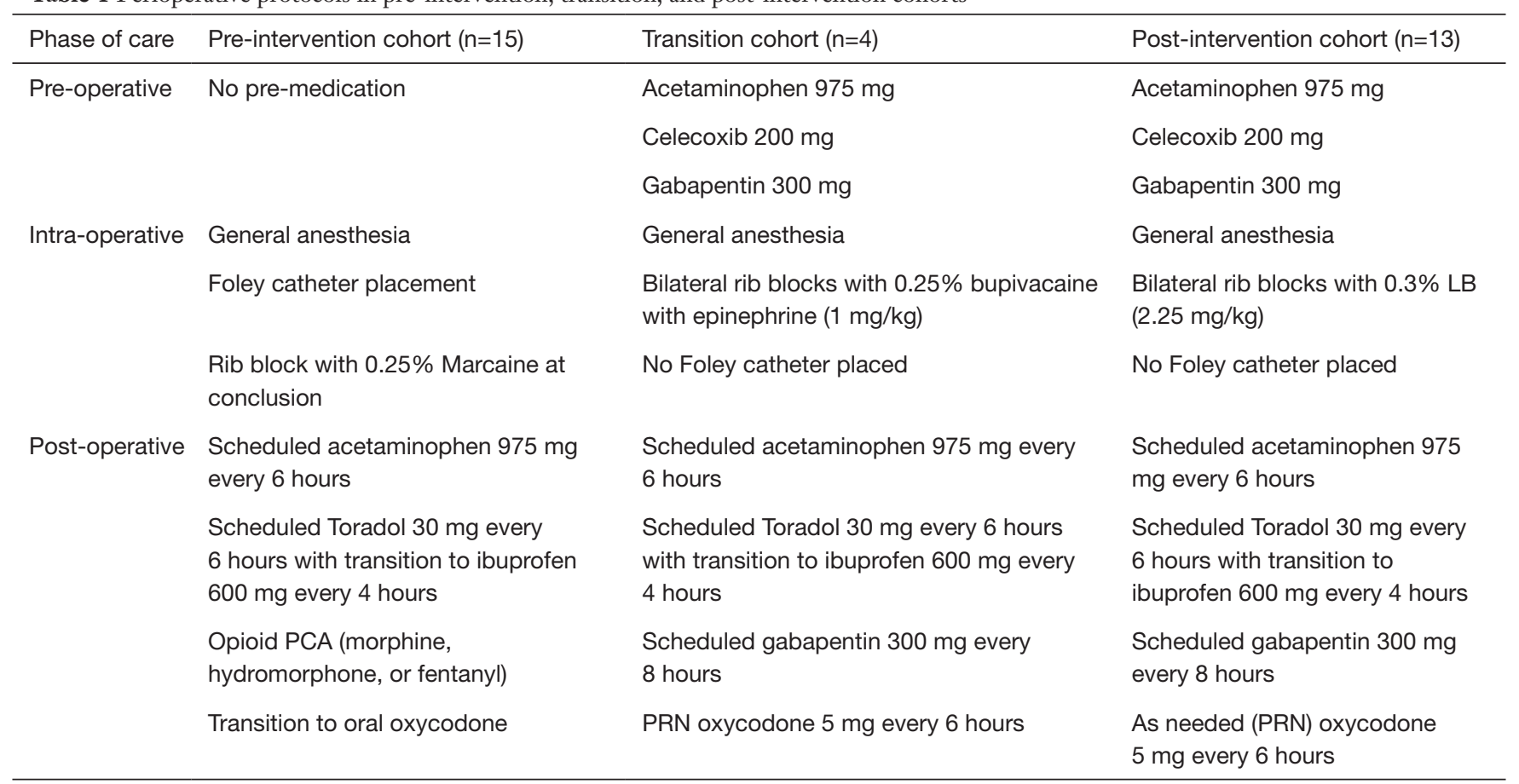

LB, liposomal bupivacaine; PCA, patient-controlled analgesia; PRN, as-needed.

previously described at our institution (9). The block is performed immediately following scope insertion at the beginning of the case, with percutaneous injection under direct thoracoscopic visualization on the right side and localized by anatomic landmarks on the left. Patients in the transition group were administered $0.25 \%$ bupivacaine with epinephrine $(1 \mathrm{mg} / \mathrm{kg})$, and patients in the post-intervention cohort were administered $1.3 \% \mathrm{LB}(2.25 \mathrm{mg} / \mathrm{kg})$ diluted in normal saline. Initially LB was not approved for patients under age 18 at our institution; approval was later granted for use in ages $13-18$, and all subsequent Nuss patients were treated with $\mathrm{LB}$.

The surgical technique was otherwise unchanged between cohorts. We do not use a sternal elevator. If one bar was placed, one stabilizer was used on the nondominant hand side. If two bars were placed, two stabilizers were used, one on each bar, on opposite sides.

Post-operatively, patients in the transition and postintervention cohorts were treated with scheduled acetaminophen, NSAIDs (IV ketorolac then oral ibuprofen), and scheduled gabapentin. Oral oxycodone was prescribed as needed only.

Patients in all groups were instructed in the use of an incentive spirometer on postoperative day 0 and got out of bed with physical therapy on postoperative day 1 . Patients in the transition and post-intervention groups did not have Foley catheters placed intraoperatively. This was routinely performed in the pre-intervention group. All patients were discharged home with a prescription for 10 tablets of oral oxycodone $5 \mathrm{mg}$ and a 30-day supply of gabapentin. Patients were counseled at discharge on as-needed use of Tylenol and Ibuprofen for mild pain in the immediate postoperative period.

Data collected from the medical record included demographics, pre-operative characteristics including Haller Index and presence or absence of cardiac or pulmonary compression, medication administration, presence of postoperative pneumothorax, and postoperative complications including need for readmission. Pain scores (rated 1-10) were documented every 4 hours by the bedside nurse. The minimum and maximum pain score for each 24-hour period (0-24 hours, 25-48 hours, and 49-72 hours postoperatively) were collected. Medication administration and dosing were collected from the intraoperative anesthesia record and the medication administration record for the hospital stay. Data collected on opioid medication dosing was converted to median morphine equivalent milligrams/kilogram (MME $\mathrm{mg} / \mathrm{kg}$ ) for standardized comparison. 
Table 2 Patient demographic and clinical characteristics in pre-intervention, transition, and post-intervention cohorts

\begin{tabular}{|c|c|c|c|c|}
\hline Patient characteristic & Pre-intervention cohort $(n=15)$ & Transition cohort $(n=4)$ & Post-intervention cohort $(n=13)$ & $P$ value \\
\hline Weight at operation (kg), mean (SD) & $57.8(8.8)$ & $57.5(5.4)$ & $66.3(11.1)$ & 0.02 \\
\hline Sex, female, $n(\%)$ & $5(33.3)$ & $1(25.0)$ & $0(0)$ & \\
\hline ASA Score, median (IQR) & $1[1,2]$ & $1[1,1.5]$ & $2[1,2]$ & 0.276 \\
\hline Cardiac dysrhythmia, n (\%) & $0(0)$ & $0(0)$ & $1(7.6)$ & \\
\hline Mitral value prolapse, $\mathrm{n}(\%)$ & $0(0)$ & $0(0)$ & $1(7.6)$ & \\
\hline Second bar placed, n (\%) & $4(26.7)$ & $1(25.0)$ & $2(15.4)$ & \\
\hline
\end{tabular}

ASA Score, American Society of Anesthesiologists Score; IQR, interquartile range; SD, standard deviation.

\section{Statistical analysis}

Statistical data analysis was performed using SAS version 9.4 (Cary, North Carolina). The primary outcomes assessed were opioid administration (MME $\mathrm{mg} / \mathrm{kg}$ ) and postoperative pain control. Secondary outcomes included postoperative clinical variables including hospital length of stay and clinical safety profile. Associations between continuous variables were examined with the Wilcoxon Rank-Sum test for non-normally distributed data, and the Student's $t$-test for normally distributed data. Chi-Square test was used to analyze categorical variables. The results expressed are a comparison between the pre-intervention and postintervention cohorts; data from the transition cohort is presented for context but is excluded from the final comparative analysis. Normally distributed data is expressed as mean \pm standard deviation (SD). Non-parametric data is expressed as median and interquartile ranges. A P value of $<0.01$ was considered statistically significant, to account for multi-comparison bias. We additionally utilized $\mathrm{X}$-mR process control charts to demonstrate time trends in our primary outcome of post-operative opioid use. The centerline and upper confidence levels are fixed after the first 10 points of our pre-intervention cohort. This allowed the determination if a significant shift occurred following our intervention. The post-operative opioid use $\mathrm{X}$-mR process control chart was created using the QIMacros package (Denver, Colorado) in Microsoft Excel 2016 (Redmond, Washington).

\section{Ethics approval}

The study was conducted in accordance with the
Declaration of Helsinki (as revised in 2013). The study was reviewed by institutional/regional/national ethics/ committee/ethics board of the University of Virginia (No. 00006183) and individual consent for this retrospective analysis was waived, as this protocol was deemed exempt as part of an institutional quality improvement initiative.

\section{Results}

A total of thirty-two patients undergoing the Nuss procedure between 2013 and 2021 at our institution were included in the study. There were no significant differences in baseline demographic and clinical characteristics between groups (Table 2). There were no significant differences in the Haller index or degree of cardiac or pulmonary compression between groups pre-operatively. The total postoperative median MMEs were markedly decreased in the post-intervention cohort ( 0.8 vs. $8.2 \mathrm{MME} \mathrm{mg/kg}$, $\mathrm{P}<0.0001$ ), with patients in this group receiving $90 \%$ fewer opioids compared to the pre-intervention cohort. Figure 1 demonstrates the $\mathrm{X}-\mathrm{mR}$ process control chart for postoperative opioid administration. The chart demonstrates a marked inflection point corresponding with our intervention. Mean total NSAIDs used during admission were similar in the pre-intervention and post-intervention groups $(2,600$ vs. 2,008.3 $\mathrm{mg}$ ibuprofen and 249.5 vs. $227 \mathrm{mg}$ Toradol), with an additional single dose of celecoxib pre-operatively in the post-intervention cohort. There were no significant differences in post-operative patient-reported pain scores between groups at 24, 48 and 72 hours (Table 3, Figure 2).

Postoperative length of stay was decreased in the postintervention cohort, with a mean length of stay (LOS) of 
Xm Chart of Post-Operative Morphine Equivalents $(\mathrm{mg} / \mathrm{kg})$
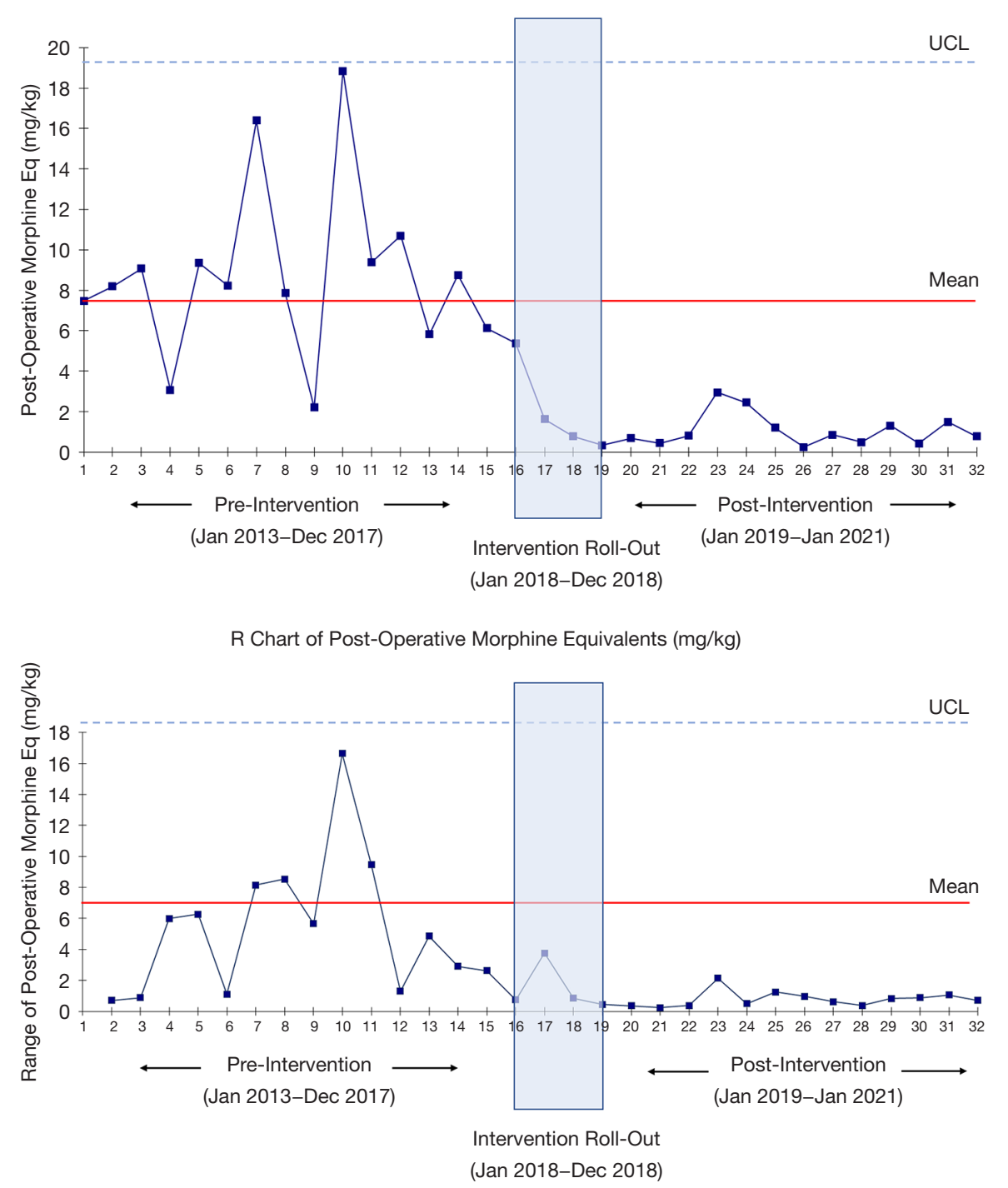

Figure 1 Process-control chart of post-operative morphine equivalents (MME $\mathrm{mg} / \mathrm{kg}$ ). Each point on the X-axis represents an individual patient, and patients are displayed in chronologic order. The blue box represents the transition cohort group (date range, Jan-Dec 2018). UCL, upper control limit; MME, median morphine equivalent.

3 days (range, 2-5 days), compared to 4 days (range, 3-6 days) in the pre-intervention cohort $(\mathrm{P}=0.002)$. Additionally, the safety profiles between the cohorts were statistically similar (Table 4). No patients required readmission for pain, and there was no incidence of bupivacaine toxicity. While a small residual pneumothorax is common following the thoracoscopic procedure, no patient required intervention for pneumothorax or hemothorax. One patient in the postintervention cohort required re-operation one year after the initial procedure due to a granulomatous reaction to the fiber wires. One patient in the post-intervention cohort developed a surgical site infection 5 months after his operation, requiring antibiotic therapy and fluid aspiration in clinic. There were no major complications (sepsis, unplanned reintubation, un-planned re-operation) during the initial hospital stay of the studied patients.

The patients in all cohorts tolerated the procedure without the development of chronic pain. No patient required a refill of oxycodone after discharge, and no patient in any cohort remained on opioid therapy 30 days after surgery. One 
Table 3 Pain control data in pre-intervention, roll-out and post-intervention cohorts

\begin{tabular}{|c|c|c|c|c|}
\hline Pain control category & $\begin{array}{l}\text { Pre-intervention cohort } \\
\qquad(n=15)\end{array}$ & $\begin{array}{l}\text { Transition cohort } \\
\qquad(\mathrm{n}=4)\end{array}$ & $\begin{array}{l}\text { Post-intervention cohort } \\
\qquad(n=13)\end{array}$ & $P$ value \\
\hline $\begin{array}{l}\text { Intra-operative median morphine } \\
\text { equivalents (mg/kg) median (IQR) }\end{array}$ & $1.4(0.7,2.5)$ & $1.1(0.1,2.3)$ & $0.4(0.1,1.4)$ & 0.022 \\
\hline $\begin{array}{l}\text { Post-operative median morphine } \\
\text { equivalents (mg/kg) median (IQR) }\end{array}$ & $8.2(6.1,9.4)$ & $1.2(0.6,3.5)$ & $0.8(0.5,1.3)$ & $<0.0001$ \\
\hline Post-operative gabapentin (g), mean (SD) & $0(0)$ & $3.38(1.79)$ & $3.0(1.3)$ & \\
\hline $\begin{array}{l}\text { Post-operative PO acetaminophen (g), } \\
\text { median (IQR) }\end{array}$ & $7.3(4.5,9.7)$ & $9.7(9.7,11.9)$ & $10.7(7.8,12.2)$ & 0.032 \\
\hline \multicolumn{5}{|l|}{ Pain score } \\
\hline 0-24 hours (maximum), median [IQR] & $8[7,10]$ & $5[3,8.5]$ & $8.5[7,10]$ & 0.535 \\
\hline 24-48 hours (maximum), median [IQR] & $7[5,8]$ & $6[4.5,7]$ & $6.5[6,8]$ & 0.689 \\
\hline 48-72 hours (maximum), median [IQR] & $6[5,8]$ & $7[5.5,8.5]$ & $6[4.5,8]$ & 0.675 \\
\hline
\end{tabular}

$\mathrm{IQR}$, interquartile range; SD, standard deviation; $\mathrm{PO}$, by mouth.

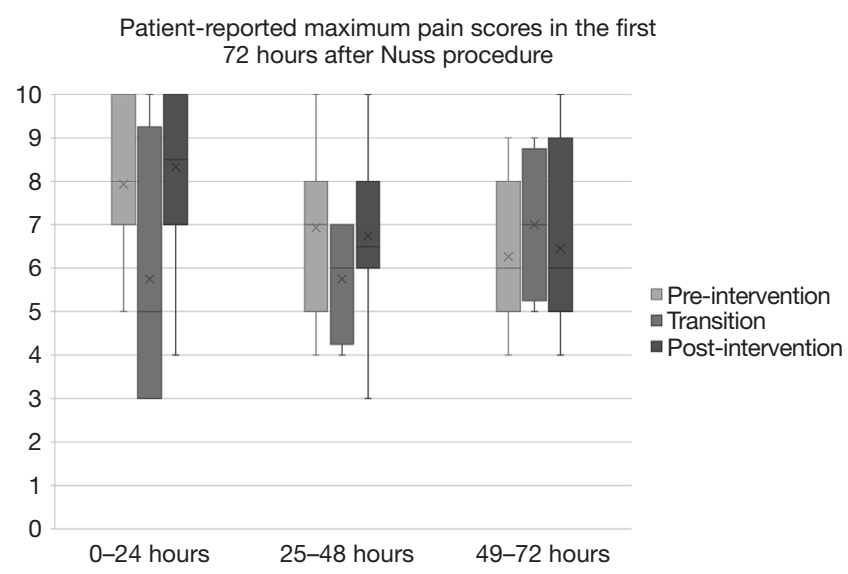

Figure 2 Patient-reported maximum pain scores in the first 72 hours after Nuss procedure

patient in the post-intervention group did require a refill of gabapentin in the post-operative period, but was able to wean off of this without need for long-term use.

\section{Discussion}

By implementing a new ERP with a multimodal pain protocol including intercostal blocks with LB and the elimination of opioid PCA use we decreased opioid utilization by $90 \%$ and decreased postoperative length of stay by one full day among adolescent patients undergoing the Nuss procedure for pectus excavatum. In our institutional protocol, we employed multiple evidencebased strategies to manage postoperative pain. Our protocol was modeled after many elements of the University of Virginia adult Thoracic Surgery ERP, which incorporates pre-operative education, opioid-sparing analgesia, rib blocks with LB, and early ambulation (19). There is a growing interest in ERP and fast-track protocols in pediatric surgery, with the aims to minimize the physiologic and psychological stress of surgery, improve patient experience and decrease hospital length of stay $(2,20-23)$.

A number of ERP and Early Recovery after Surgery (ERAS) protocols have been published for the Nuss procedure. Holmes et al. noted a decrease in hospital length of stay, patient-reported pain scores, and opioid consumption among pediatric Nuss patients with the implementation of a multimodal pain regimen that included placement of a paravertebral pain catheter for analgesia (24). Litz et al. similarly reported decreased hospital length of stay and postoperative morphine equivalents following 
Table 4 Operative time and post-operative length of stay in pre-intervention, roll-out and post-intervention cohorts

\begin{tabular}{lccc}
\hline Clinical parameter & $\begin{array}{c}\text { Pre-intervention cohort } \\
(\mathrm{n}=15)\end{array}$ & $\begin{array}{c}\text { Transition cohort } \\
(\mathrm{n}=4)\end{array}$ & $\begin{array}{c}\text { Post-intervention cohort } \\
(\mathrm{n}=13)\end{array}$ \\
\hline $\begin{array}{l}\text { Post-operative LOS (days) } \\
\text { median [IQR] }\end{array}$ & $4[3,5]$ & $3[3,4.5]$ & $3[2.5,3]$ \\
$\begin{array}{l}\text { Length of case (hours intubated } \\
\text { intra-operatively) mean (SD) }\end{array}$ & $3.5(0.6)$ & $3.5(0.9)$ & $3.5(0.4)$ \\
\hline
\end{tabular}

LOS, length of stay, n, number of patients; IQR, interquartile range; SD, standard deviation.

implementation of a protocol involving pre-operative expectation setting and multimodal analgesia, with use of an opioid PCA (20). Wharton and colleagues described a multimodal ERAS protocol involving bilateral multi-level intercostal nerve blocks with $0.25 \%$ Marcaine during the Nuss procedure (22). As part of their protocol, patients were prescribed stretches and were treated postoperatively with gabapentin and opioid and ketamine PCAs. A decrease in hospital length of stay and urinary retention was noted with these interventions, with decreased patient-reported pain scores on post-operative day zero.

There is also a growing interest in INC for pain control following the Nuss procedure. Graves et al. found that in comparison to thoracic epidural analgesia, patients undergoing cryoanalgesia had a significantly decreased opioid requirement while inpatient, and length of stay was decreased from 5 to 3 days in the INC group (14). In a small study by Rettig et al. same-day discharge was possible for $66.7 \%$ of patients who underwent a combination of INC and intercostal nerve block (15). This modality certainly shows promise, though Sun et al. noted an increased rate of slipped bars requiring reoperation among patients undergoing INC [OR 36.65 (5.04-266.39)] (16). Disadvantages of this technique include the significant expense and need for training associated with the use of this specialized equipment.

The protocol we have implemented incorporates existing evidence-based strategies for postoperative pain control and early recovery, augmented by the addition of the use of LB for longer-lasting local analgesia in the perioperative period, which has a duration of 1-4 days (25). In addition to early mobilization and elimination of urinary catheter placement, a major tenet of our protocol is to address pain in the preoperative, intraoperative, and postoperative phases.

Preoperative administration of gabapentin (26) and anti-inflammatories have been shown to decrease the inflammatory response to surgery and the need for postoperative opioid medication. Multimodal preemptive analgesia has been shown to decrease hyperalgesia and peripheral and central pain sensitization, particularly in combination with a continued multimodal pain strategy postoperatively (27). For this reason, we treat patients preoperatively with single doses of gabapentin, acetaminophen, and celecoxib.

Bilateral intercostal blocks are performed by the surgeon as soon as the thoracoscope is inserted. This provides local analgesia similar to that seen with paravertebral blocks or cryoablation, but is a technique readily learned and performed by surgeons and does not require specialized equipment. The addition of this step did not significantly increase operative time among our patients. Intercostal block with standard bupivacaine has previously been shown to decrease opioid consumption in pediatric patients undergoing the Nuss procedure (12). Compared to standard bupivacaine, rib block with LB has been shown in the adult population to decrease opioid administration and length of stay following thoracoscopic surgery when used as part of a multimodal early recovery program, with decreased pain scores on postoperative days 0-2 (28) and 0-3 (29). Findings on the use of LB for intercostal blocks outside the context of a multimodal protocol in adult thoracic surgery patients are mixed, with a recent small clinical trial reporting no difference in pain control and opioid use between cohorts undergoing rib block with LB compared to bupivacaine with epinephrine (30). Studies have shown decreased opioid consumption with the utilization of LB in pediatric spine surgery $(31,32)$, but little has been previously published about the use of LB in adolescents undergoing thoracic surgery. Though interpretation is limited greatly by the small size of the transition cohort, pain control appeared to be similar between the transition (ERP using bupivacaine with epinephrine) and post-intervention (ERP using LB) cohorts. This, as well as the multimodal nature of the intervention, makes it difficult to conclude that the addition of LB alone is responsible for the significant decrease in opioid consumption in this study, but is more likely one of 
several factors contributing to this outcome.

Postoperatively, patients in the post-intervention cohort received as-needed oral oxycodone, but opioid requirements were significantly lower than those in patients who were given a PCA, without a difference in pain scores. While decreased opioid use in the postoperative phase is likely multifactorial and much can be attributed to improved pain control from the pre- and intra-operative interventions, the change from routine PCA use to as-needed oral opiates may also be an important factor. Logan and Rose demonstrated that PCA use and pain scores were significantly higher in adolescent patients who reported preoperative anxiety and high expected levels of postoperative pain (33). Similarly, Schlatter et al. achieved a significant decrease in postoperative length of stay by implementing a protocol incorporating intercostal nerve blocks, scheduled oral analgesia, and preoperative consultation aimed at patient education about anxiety and expectations for pain control (34). The healthcare team and parents may additionally contribute to PCA overuse by encouraging the child to "stay ahead of the pain", leading to self-dosing at a more frequent interval than is actually needed. By removing the option to frequently self-dose, restricting access to opioid medication to as-needed dosing every 6 hours may decrease the misuse of this medication to treat patient or family anxiety or anticipatory pain.

Adjunct measures to support early recovery included early ambulation (out of bed with Physical Therapy on postoperative day 1) and elimination of routine Foley catheter placement. Based on experience with the adult Thoracic Surgery ERAS program at our institution, we found routine urinary catheter placement was not necessary. Though the mobilization protocol was consistent between all groups, improved pain control and lack of a urinary catheter may contribute to increased early mobility and decreased length of stay.

Limitations of this study included the small cohort sizes, retrospective nature of the comparison group and that it was conducted at a single institution. As the study included a retrospective pre-intervention group, there was some variability in the exact medications administered in this group (fentanyl vs. hydromorphone, occasional cyclobenzaprine). Uniformity of medication administration was greatly improved in the post-intervention cohort once the protocol was put in place. It is important to note that multiple interventions were put into place at once with the implementation of the protocol. This limits our ability to determine the impact of individual elements within the protocol (for example, introducing rib blocks versus replacement of PCA analgesia with oral opioids) on the outcomes observed. Despite the size of the single institution cohorts, we could see a statistically and clinically significant impact of our intervention on opioid administration.

\section{Conclusions}

A significant decrease in opioid use and hospital length of stay after the Nuss procedure were achieved by the implementation of a multi-modal protocol including LB for rib blocks for pain management, without an increase in patient-reported pain scores. The procedural rib block and post-operative pain management protocol described in this investigation are straightforward and could be easily implemented at other institutions.

\section{Acknowledgments}

This research has been presented as a digital poster presentation at the 2021 meeting of the International Pediatric Endosurgery Group, June 2021.

Funding: None.

\section{Footnote}

Reporting Checklist: The authors have completed the TREND reporting checklist. Available at https://dx.doi. org/10.21037/jtd-21-1314

Data Sharing Statement: Available at https://dx.doi. org/10.21037/jtd-21-1314

Conflicts of Interest: All authors have completed the ICMJE uniform disclosure form (available at https://dx.doi. org/10.21037/jtd-21-1314). LWM is a consultant for Astra Zeneca (use of Osimertinib in resected lung cancer), On Target Laboratories, Pacira Pharmaceuticals (not active for $>12$ months prior to submission of this manuscript). The other authors have no conflicts of interest to declare.

Ethical Statement: The authors are accountable for all aspects of the work in ensuring that questions related to the accuracy or integrity of any part of the work are appropriately investigated and resolved. The study was conducted in accordance with the Declaration of Helsinki (as revised in 2013). The study was reviewed by institutional/ regional/national ethics/committee/ethics board of the University of Virginia (No. 00006183) and individual 
consent for this retrospective analysis was waived, as this protocol was deemed exempt as part of an institutional quality improvement initiative.

Open Access Statement: This is an Open Access article distributed in accordance with the Creative Commons Attribution-NonCommercial-NoDerivs 4.0 International License (CC BY-NC-ND 4.0), which permits the noncommercial replication and distribution of the article with the strict proviso that no changes or edits are made and the original work is properly cited (including links to both the formal publication through the relevant DOI and the license). See: https://creativecommons.org/licenses/by-nc-nd/4.0/.

\section{References}

1. Nuss D, Kelly RE Jr, Croitoru DP, et al. A 10-year review of a minimally invasive technique for the correction of pectus excavatum. J Pediatr Surg 1998;33:545-52.

2. Muhly WT, Maxwell LG, Cravero JP. Pain management following the Nuss procedure: a survey of practice and review. Acta Anaesthesiol Scand 2014;58:1134-9.

3. Singhal NR, Jerman JD. A review of anesthetic considerations and postoperative pain control after the Nuss procedure. Semin Pediatr Surg 2018;27:156-60.

4. Densmore JC, Peterson DB, Stahovic LL, et al. Initial surgical and pain management outcomes after Nuss procedure. J Pediatr Surg 2010;45:1767-71.

5. Allison CE, Aronson DC, Geukers VG, et al. Paraplegia after thoracotomy under combined general and epidural anesthesia in a child. Paediatr Anaesth 2008;18:539-42.

6. Kelly RE Jr, Obermeyer RJ, Goretsky MJ, et al. Recent Modifications of the Nuss Procedure: The Pursuit of Safety During the Minimally Invasive Repair of Pectus Excavatum. Ann Surg 2020. [Epub ahead of print]. doi: 10.1097/SLA.0000000000003877.

7. Kolvekar S, Pilegaard H, Ashley E, et al. Pain management using patient controlled anaesthesia in adults post Nuss procedure: an analysis with respect to patient satisfaction. J Vis Surg 2016;2:37.

8. Dolin SJ, Cashman JN. Tolerability of acute postoperative pain management: nausea, vomiting, sedation, pruritus, and urinary retention. Evidence from published data. $\mathrm{Br} \mathrm{J}$ Anaesth 2005;95:584-91.

9. Martin LW, Mehran RJ. Intercostal nerve blockade for thoracic surgery with liposomal bupivacaine: the devil is in the details. J Thorac Dis 2019;11:S1202-5.

10. Thiele RH, Rea KM, Turrentine FE, et al. Standardization of care: impact of an enhanced recovery protocol on length of stay, complications, and direct costs after colorectal surgery. J Am Coll Surg 2015;220:430-43.

11. Bryskin RB, Robie DK, Mansfield FM, et al. Introduction of a novel ultrasound-guided extrathoracic sub-paraspinal block for control of perioperative pain in Nuss procedure patients. J Pediatr Surg 2017;52:484-91.

12. Lukosiene L, Macas A, Trepenaitis D, et al. Single shot intercostal block for pain management in pediatric patients undergoing the Nuss procedure: a doubleblind, randomized, controlled study. J Pediatr Surg 2014;49:1753-7.

13. Luo M, Liu X, Ning L, et al. Comparison of Ultrasonography-guided Bilateral Intercostal Nerve Blocks and Conventional Patient-controlled Intravenous Analgesia for Pain Control After the Nuss Procedure in Children: A Prospective Randomized Study. Clin J Pain 2017;33:604-10.

14. Graves CE, Moyer J, Zobel MJ, et al. Intraoperative intercostal nerve cryoablation During the Nuss procedure reduces length of stay and opioid requirement: A randomized clinical trial. J Pediatr Surg 2019;54:2250-6.

15. Rettig RL, Rudikoff AG, Lo HYA, et al. Same day discharge for pectus excavatum-is it possible? J Pediatr Surg 2021. [Epub ahead of print]. doi: 10.1016/ j.jpedsurg.2021.02.007.

16. Sun RC, Mehl SC, Anbarasu CR, et al. Intercostal cryoablation during Nuss procedure: A large volume single surgeon's experience and outcomes. J Pediatr Surg 2021. [Epub ahead of print]. doi: 10.1016/j.jpedsurg.2021.03.006.

17. Keller BA, Kabagambe SK, Becker JC, et al. Intercostal nerve cryoablation versus thoracic epidural catheters for postoperative analgesia following pectus excavatum repair: Preliminary outcomes in twenty-six cryoablation patients. J Pediatr Surg 2016;51:2033-8.

18. Rettig RL, Rudikoff AG, Lo HYA, et al. Cryoablation is associated with shorter length of stay and reduced opioid use in pectus excavatum repair. Pediatr Surg Int 2021;37:67-75.

19. Martin LW, Sarosiek BM, Harrison MA, et al. Implementing a Thoracic Enhanced Recovery Program: Lessons Learned in the First Year. Ann Thorac Surg 2018;105:1597-604.

20. Litz CN, Farach SM, Fernandez AM, et al. Enhancing recovery after minimally invasive repair of pectus excavatum. Pediatr Surg Int 2017;33:1123-9.

21. Reismann M, Arar M, Hofmann A, et al. Feasibility of fast-track elements in pediatric surgery. Eur J Pediatr Surg 
2012;22:40-4.

22. Wharton K, Chun Y, Hunsberger J, et al. Successful use of an enhanced recovery after surgery (ERAS) pathway to improve outcomes following the Nuss procedure for pectus excavatum. J Pediatr Surg 2020;55:1065-71.

23. Yu P, Wang G, Zhang C, et al. Clinical application of enhanced recovery after surgery (ERAS) in pectus excavatum patients following Nuss procedure. J Thorac Dis 2020;12:3035-42.

24. Holmes DM, Polites SF, Roskos PL, et al. Opioid use and length of stay following minimally invasive pectus excavatum repair in 436 patients - Benefits of an enhanced recovery pathway. J Pediatr Surg 2019;54:1976-83.

25. Manson WC, Blank RS, Martin LW, et al. An Observational Study of the Pharmacokinetics of SurgeonPerformed Intercostal Nerve Blockade With Liposomal Bupivacaine for Posterior-Lateral Thoracotomy Analgesia. Anesth Analg 2020;131:1843-9.

26. Ho KY, Gan TJ, Habib AS. Gabapentin and postoperative pain--a systematic review of randomized controlled trials. Pain 2006;126:91-101.

27. Vadivelu N, Mitra S, Schermer E, et al. Preventive analgesia for postoperative pain control: a broader concept. Local Reg Anesth 2014;7:17-22.

28. Patel KM, van Helmond N, Kilzi GM, et al. Liposomal Bupivacaine Versus Bupivacaine for Intercostal Nerve Blocks in Thoracic Surgery: A Retrospective Analysis. Pain Physician 2020;23:E251-8.

29. Kodia K, Razi SS, Stephens-McDonnough JA, et al. Liposomal Bupivacaine Versus Bupivacaine/Epinephrine

Cite this article as: Eubanks AL, Grabski DF, Pollack J, Levin DE, McGahren E, Martin LW, Gander J. A multimodal protocol utilizing liposomal bupivacaine rib blocks leads to opioid reduction in patients undergoing the Nuss procedure. $\mathrm{J}$ Thorac Dis 2021;13(11):6363-6372. doi: 10.21037/jtd-21-1314
Intercostal Nerve Block as Part of an Enhanced Recovery After Thoracic Surgery (ERATS) Care Pathway for Robotic Thoracic Surgery. J Cardiothorac Vasc Anesth 2021;35:2283-93.

30. Weksler B, Sullivan JL, Schumacher LY. Randomized trial of bupivacaine with epinephrine versus bupivacaine liposome suspension in patients undergoing minimally invasive lung resection. J Thorac Cardiovasc Surg 2021;161:1652-61.

31. Chughtai M, Sultan AA, Hudson B, et al. Liposomal Bupivacaine Is Both Safe and Effective in Controlling Postoperative Pain After Spinal Surgery in Children: A Controlled Cohort Study. Clin Spine Surg 2020;33:E533-8.

32. Ballock RT, Seif J, Goodwin R, et al. Clinical and Economic Outcomes Associated With Use of Liposomal Bupivacaine Versus Standard of Care for Management of Postsurgical Pain in Pediatric Patients Undergoing Spine Surgery. J Health Econ Outcomes Res 2021;8:29-35.

33. Logan DE, Rose JB. Is postoperative pain a self-fulfilling prophecy? Expectancy effects on postoperative pain and patient-controlled analgesia use among adolescent surgical patients. J Pediatr Psychol 2005;30:187-96.

34. Schlatter MG, Nguyen LV, Tecos M, et al. Progressive reduction of hospital length of stay following minimally invasive repair of pectus excavatum: A retrospective comparison of three analgesia modalities, the role of addressing patient anxiety, and reframing patient expectations. J Pediatr Surg 2019;54:663-9. 